\title{
Dramatic Responses of Recurrent Upper Urinary Tract Urothelial Carcinoma Harboring FGFR3 and TP53 Activating Mutations to Pembrolizumab in Combination with Erdafitinib: A Case Report
}

This article was published in the following Dove Press journal: OncoTargets and Therapy

\author{
Xinjia Ding' \\ Jianguo Zong ${ }^{2}$ \\ Xiang $\mathrm{Li}^{2}$ \\ Xiaoyan $\mathrm{Bai}^{2}$ \\ Bowen $\operatorname{Tan}^{2}$ \\ Weibing Sun (1D) \\ Ruoyu Wang ${ }^{2}$ \\ Yan Ding (D) ${ }^{2,3}$ \\ 'Department of Urology, The Affiliated \\ Zhongshan Hospital of Dalian University, \\ Dalian, I I600I, People's Republic of \\ China; ${ }^{2}$ The Institute for Translational \\ Medicine, The Affiliated Zhongshan \\ Hospital of Dalian University, Dalian, \\ I I600I, People's Republic of China; \\ ${ }^{3}$ Department of Pediatrics, Boston \\ Children's Hospital, Harvard Medical \\ School, Boston, MA, 021I5, USA
}

Correspondence: Yan Ding

Department of Pediatrics, Boston

Children's Hospital, Harvard Medical

School, 300 Longwood Ave, Boston, MA,

20II5, USA

Tel $+\mid$ 617-642-1698

Fax + 6|7-73|-0787

Email yan.ding@childrens.harvard.edu

Ruoyu Wang

Email wangruoyu1963@163.com
Background: Upper tract urothelial carcinoma (UTUC) has a high recurrence rate and is likely refractory to systemic chemotherapy. The long-term outcomes and responses to immunotherapy and retreatment regimen after tumor recurrence for such cases had not yet been well-documented.

Case Presentation: Here we report a unique case of long-term follow-up with a 67 -yearold woman, who was diagnosed with advanced UTUC, received radical nephroureterectomy with bladder cuff, and was refractory to chemotherapy with cisplatin and gemcitabine. Positive PD-L1 expression and somatic mutation of Ser249Cys in FGFR3 were identified in the tumor tissue. The patient then received pembrolizumab monotherapy and achieved complete response (CR) after 6 cycles of treatment. She discontinued pembrolizumab treatment thereafter but remained in CR for 3 years and 7 months until the recurrence of tumor in the right mid-ureter. The patient was then retreated with a combination of pembrolizumab and erdafitinib, and achieved CR again after the third cycle of treatment.

Conclusion: We reported here a rare case of UTUC with concurrent pathogenic mutations in FGFR3 and TP53 with positive PD-L1 expression. The patient archived exceptional therapeutic responses to PD-1 blockade treatment and retreatment with combination of pembrolizumab and erdafitinib. Our results provide new insight into the duration of immunotherapy and the retreatment strategy after tumor recurrence based on individual genomic profiles.

Keywords: upper tract urothelial carcinoma, PD-1 blockade, pembrolizumab, immunotherapy, erdafitinib

\section{Introduction}

Bladder carcinoma (BC) is the most common tumor of the urinary system and the seventh most prevalent cancer globally. Urothelial carcinoma (UC) accounts for $90 \%$ of the $\mathrm{BC}$, which is originated from the bladder, renal pelvis, and/or ureter. ${ }^{1,2}$ Worldwide, UC caused approximately 165,000 deaths each year, and the 5-year survival rate is about $5 \%$ in cases of metastasis. ${ }^{3,4}$ UCs can be subdivided into lower tract urothelial carcinomas (LTUC) mainly from bladder and urethra, and upper tract urothelial carcinomas (UTUC) located in the pyelocaliceal cavity and ureter. ${ }^{1}$ While surgery is the first line of treatment for early and mid-staged UC, patients with advanced UTUCs usually receive combination of cisplatin and 
gemcitabine as the first-line chemotherapy. Although the two primary treatment options have achieved high response rate, the overall prognosis remains poor. ${ }^{5,6}$ Majority patients with late-stage UTUC experience intravesical recurrence, lymph node, and other systemic metastases within a few months or years after radical surgical resection. ${ }^{7,8}$ The common side effects of chemotherapy include renal dysfunction, leukopenia or thrombocytopenia, nausea, vomiting, and poor appetite. $^{9,10}$ Many patients with advanced UTUC refused to receive any chemotherapy due to the concerns about toxic and side effects, ${ }^{11,12}$ which highlights the unmet need for effective and tolerable first-line treatment.

PD-1 receptors have been proved to be effective therapeutic targets for various types of human cancers including UCs. $^{13-15}$ Immune checkpoint inhibitors (ICIs) such as pembrolizumab $^{16}$ and azolizumab ${ }^{17}$ have recently been assessed in patients with metastatic UCs with the objective response rate between $22 \%$ and $39 \% .{ }^{16}$ Given the high cost and potential long-lasting effect of ICIs, the optimal duration of treatment for UTUC, the length of a continuous CR after medication withdrawal, and the outcomes of retreatment either with ICI alone or a combination of ICI and targeted drug after relapse are certainly worth of investigation.

Here we report a unique case of a 67-year-old woman who was diagnosed with UTUC, received radical nephroureterectomy with bladder cuff and failed response to the routine chemotherapy with cisplatin and gemcitabine. Based on the results of PD-L1 expression level, TMB score and FGFR3 mutation profiles, the patient was initially given pembrolizumab monotherapy and achieved 3 years and 7 months of CR before the tumor recurred. The patient was then retreated with a combination of pembrolizumab and erdafitinib, a kinase inhibitor approved for the treatment of adults with locally advanced or metastatic urothelial carcinoma (mUC) which has susceptible FGFR3 or FGFR2 genetic alterations, achieved CR again after three cycles and remains progression-free until now.

\section{Case Presentation}

A 67-year-old female was admitted to the hospital with the main complaint of painless gross hematuria for one week in April 2015. CT scan showed a left mid-ureteral lesion (Figure 1A) and underwent radical nephroureterectomy with a bladder cuff on the left side in April 2015. Postoperative pathology showed invasive UC with tumor grade T2N0M0, serous membrane, renal and bladder wall tissue invasion (Figure 1B). The patient recovered well after surgery, and hematuria disappeared. Unfortunately, 6 months after the surgery, the patent's left adrenal gland and abdominal aortic lymph node enlargement were observed in the follow-up CT scan examination (Figure 2A). The patient first received chemotherapy with a combination of cisplatin
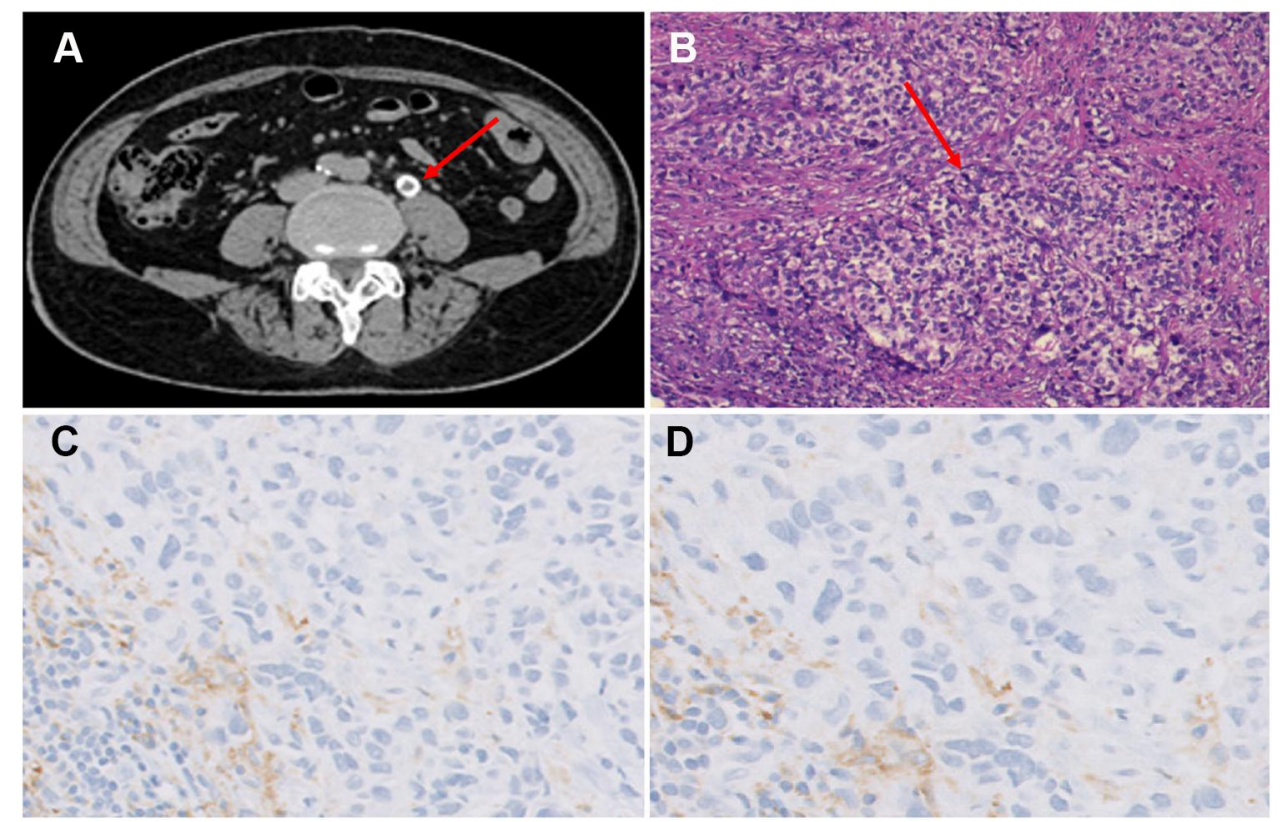

Figure I Abdomen enhancement CT scan and pathology of surgical specimen (A-D). (A) CT images showed the filling defects in the upper middle segment of the left ureter (red arrow), suggesting ureteral carcinoma. (B) 100x magnification image of the UTUC. The cancer cells were papillary, nested, densely arranged in sheets, with large and deeply stained nuclei (red arrow). H\&E stained. (C) Immunohistochemistry of PD-LI expression in the tumor tissue (DAKO 22C3). PD-LI antibody exhibited CPS $\geq 10$ at I0× magnification. (D) PD-LI antibody exhibited CPS $\geq 10$ at $20 \times$ magnification. 


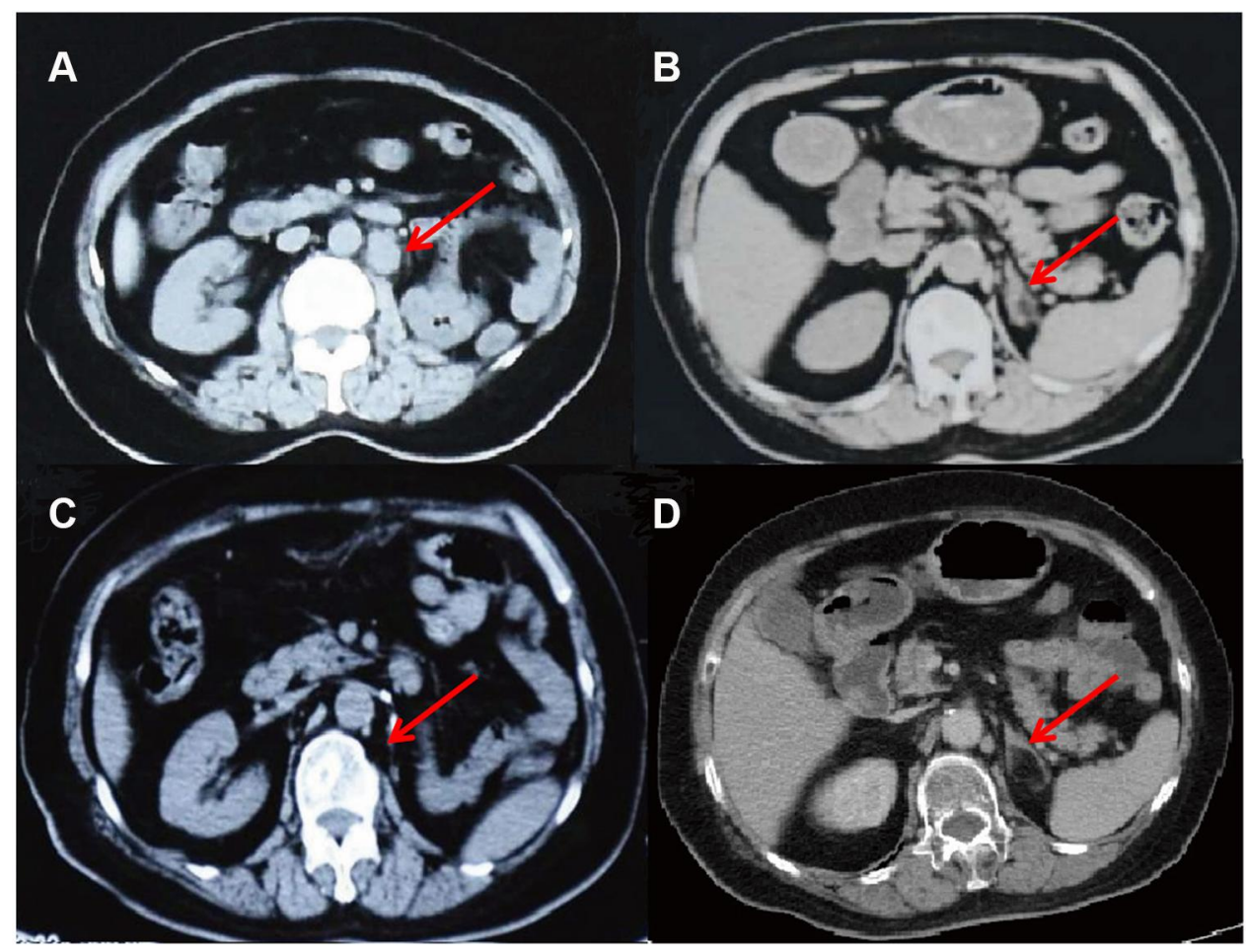

Figure 2 Abdomen CT scans after 6 months chemotherapy with cisplatin and gemcitabine ( $\mathbf{A}$ and $\mathbf{B})$ and after 6 cycles of immunotherapy with pembrolizumab (C and $\mathbf{D}$ ), images on the same sections. (A) Compared with Figure IA, the patient's left abdominal aortic lymphadenopathy was enlarged (red arrow). (B) The left main adrenal gland and medial branch nodules were thickened (red arrow), suggesting that the tumor has metastasized. (C) Abdominal aortic lymph node enlargement shrank significantly (red arrow). (D) The left main adrenal nodule disappeared (red arrow), indicating the stabilization of disease progression.

and gemcitabine for a period of 6 weeks. Gemcitabine (1000 mg/m2) was given on days 1 and 8 of each cycle. Cisplatin $(70 \mathrm{mg} / \mathrm{m} 2)$ was given on day 1 . Protocol-specified recommendations were for gemcitabine to be given as a 30 min intravenous infusion in $500 \mathrm{~mL}$ normal saline and cisplatin as a 4-h intravenous infusion in $1 \mathrm{~L}$ saline. However, she experienced nausea, anemia, diarrhea, and an apparent sign of mental depression during chemotherapy, and refused to continue the treatment. The CT scan from the follow-up visit in December 2015 showed no sign of shrinking with her swollen lymph nodes (Figure 2B).

The patient presented severe weakness and fatigue in her visit in May 2016. Given the patient's declination of chemotherapy-based palliative treatment, we decided to explore the options for potential targeted therapy and/or immunotherapy. Genetic testing of the patient surgical specimen paired with white blood cells was performed using the hybrid capture-based KnoTruth ${ }^{\text {TM }}$ OncUro-600 panel (JIEN Biotechnologies, China) on the Illumina NextSeq500 next generation sequencing (NGS) platform. The NGS data was analyzed using OncoDecoder ${ }^{\mathrm{TM}}$ (Genomic Future, Inc. USA), and cancer-specific somatic mutations in FGFR3, TP53, and CREBBP ${ }^{18}$ were identified as shown in Table 1.

The tumor harbored a pathogenic somatic missense mutation of Ser249Cys in FGFR3, hotspot Arg282Pro mutation in TP53, and deletion of Arg413* in CREBBP. ${ }^{18}$ In addition, TMB was computed with a score of 13.6. IHC was performed for PD-L1 expression using the pathological

Table I Clinical Relevant Somatic Mutation Identified in Present Case of UTUC

\begin{tabular}{|l|c|l|l|l|l|}
\hline Gene Name & Exon & Amino Acid Change & Nucleotide Change & Genotype & Mutation Type \\
\hline FGFR3 & 7 & p. Ser249Cys & c.746C>G & Heterozygous & Missense \\
\hline TP53 & 8 & p. Arg282Pro & c.845G>C & Homozygous & Missense \\
\hline CREBBP & 5 & p. Arg 413* & c. I237C>T & Heterozygous & Deletion \\
\hline
\end{tabular}

Note: *Frameshift deletion. 
specimen, and results show positive staining (Figure $1 \mathrm{C}$ and D). At the time, ICIs (eg pembrolizumab/atezolizumab) were in Phase II clinical trials. ${ }^{16}$ Pazopanib is a multikinase inhibitor that exerted an exceptional response to UTUC patient with FGFR3 Ser249Cys mutation in a case report. ${ }^{19}$ Erdafitinib, the latest FDA-approved FGFR3 inhibitor for UC in 2019, was not even available for trial at the time. ${ }^{20}$ Based on these findings, the patient was given two options of treatment regimen with either pazopanib or ICIs. Concerning the potential side effects of pazopanib in renal function and the patient's nephrectomy of the left kidney, the patient was initiated immunotherapy on pembrolizumab. The ethics committee of the Affiliated Zhongshan Hospital of Dalian University approved the study. The patient received pembrolizumab 100mg every 3 weeks for 6 cycles. The follow-up CT scans showed that the patient's left adrenal gland and abdominal aortic lymph node mass disappeared after cycle 4 of the treatment (Figure 2C and D). In contrast to the prior chemotherapy, the patient experienced only mild rash with an itch on pembrolizumab, and these symptoms improved significantly by taking mupirocin (for external use). No obvious abnormalities in renal function and liver biochemistry were observed. After 6 cycles of treatment, the drug was discontinued and pelvic CT was reviewed every 3 months, and the CR was maintained for 3 years and 7 months.

In January 2020, the patient experienced painless gross hematuria again in the follow-up visit. CT scans showed a mass lesion in the middle of the right ureter, and no other metastases in the pelvic cavity were observed (Figure 3A and B). We performed a liquid biopsy with the patient blood circulating DNA using KnoTruth ${ }^{\mathrm{TM}}$ OncUro ctDNA-UMI NGS panel (JIEN Biotechnologies, China). The results show a score of 9.3 for blood plasma TMB (bTMB), and again Ser249Cys mutation in FGFR3. Owing to the patient's sensitivity to pembrolizumab from early treatment and relatively high bTMB score in plasma ctDNA, we recommended a pembrolizumab retreatment regimen with $200 \mathrm{mg}$ every 3 weeks. However, the tumor did not show significant shrinking by the CT scan at the end of 3 cycles of treatment though the patient's hematuria was alleviated. To seek the improvement of therapy, we recommended treatment plan with a combination of pembrolizumab and erdafitinib. The 3-month follow-up CT scan results showed that the thickness of the middle wall of the right ureter was strikingly reduced, and the expansion of the right renal pelvis and effusion completely disappeared (Figure 3C and D). The patient

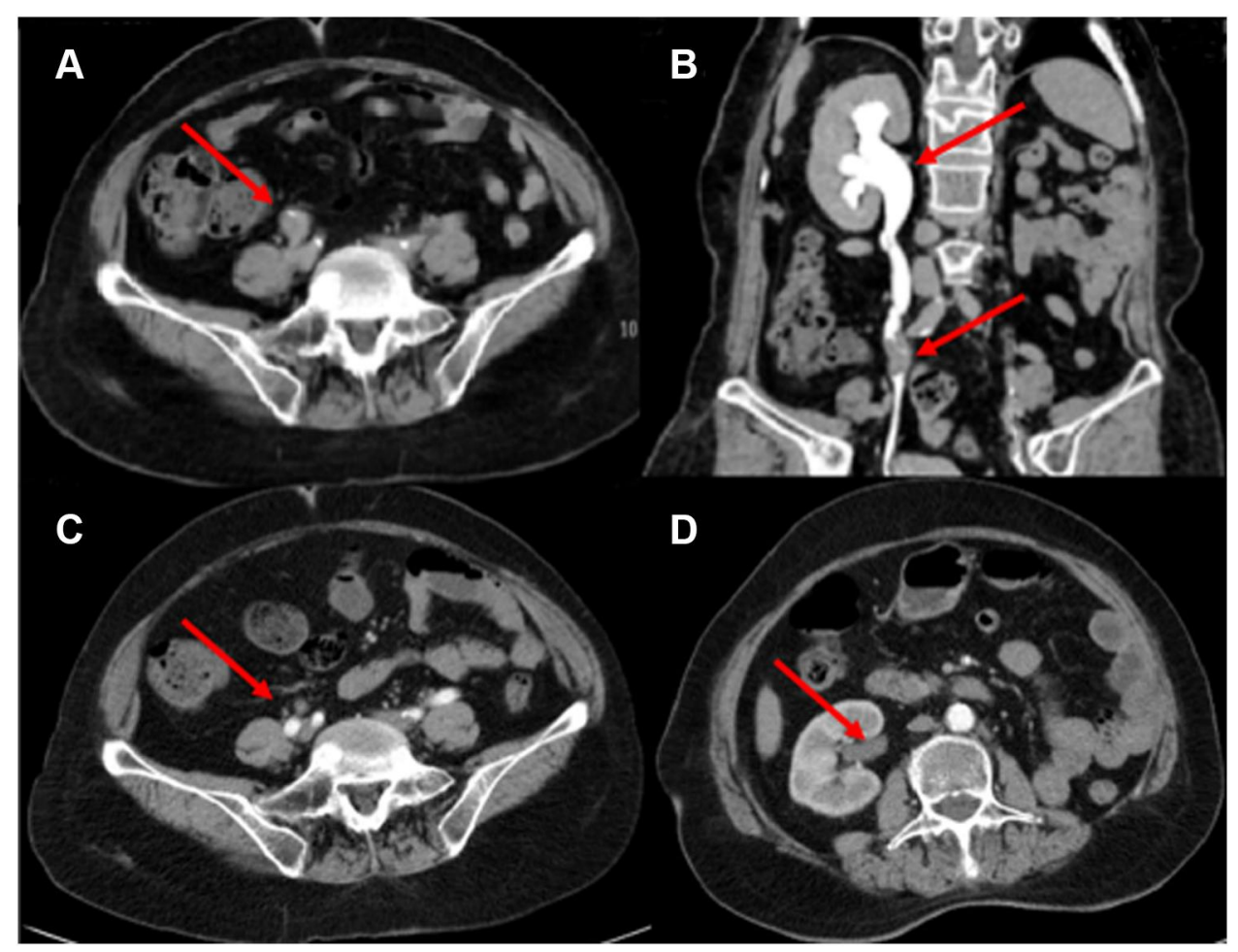

Figure 3 CT urogram. (A) Tumor recurrence in the middle right ureter (red arrow). (B) As a result of blockage in the middle ureter, the right hydronephrosis expanded (red arrow). (C) After 3 cycles of a combination of pembrolizumab and erdafitinib, the thickening of the wall of the right middle ureter was significantly reduced (red arrow). (D) The expansion of the right renal pelvis and the effusion were obviously disappeared (red arrow). 
remains progression-free at the end of 6 cycles of the combined treatment. Continuous clinical follow-up has been established for the patient.

\section{Discussion and Conclusion}

Urothelial carcinoma is the most common malignant tumor of the urinary system with high recurrence rate. ${ }^{1,2}$ Radical nephroureterectomy and partial cystectomy are commonly considered as standard the first-line treatment. In the present case, the patient presented with painless gross hematuria, left mid-ureteral lesion with multiple local invasion (Figure 1) at the initial visit. Approximately $25-40 \%$ of patients developed renal dysfunction after receiving cisplatin as the first-line chemotherapy. ${ }^{9,10}$ The severe adverse effects of chemotherapy such as anemia and neutropenia in our case often result in patient's declination or discontinuation of chemotherapies. Therefore, clinicians are always committed to finding less toxic treatment options such as targeted therapy and immunotherapy.

At the time we accepted the patient in 2016, ICIs were in phase II clinical trials. ${ }^{16}$ Reports on either targeted drug or PD-1 blockade treatment in the advanced recurrent UTUC were minimal. To seek appropriate targeted treatment or immunotherapy for the patient, we performed NGS and identified several pathogenic somatic mutations in the tumor tissue (Table 1). In addition, positive IHC of PD-L1 expression and a TMB score of 13.6 were obtained. The patient chose pembrolizumab owing to the potential side effect of pazopanib in renal function and achieved a CR. She discontinued the treatment after cycle 6 and remained $\mathrm{CR}$ for 3 years and 7 months until the recurrence of tumor in the middle of her right ureter (Figure 3). Although pembrolizumab treatment in UC was approved by the FDA two years later in 2018, the optimal duration for pembrolizumab treatment in patients with advanced UTUC is still unclear. That is because many patients discontinued such treatment due to either the high financial burden and/or concerning the risk of potential tumor nephrotoxicity of long-term treatment like the patient in our case. Recent studies showed that longterm outcomes and responses to retreatment in patients with melanoma treated with pembrolizumab. ${ }^{21}$ However, no clear guideline or practice on the duration of UC treatment with PD-1 blockade has been reported. The clinical practice in either PD-1 blockade retreatment or other alternative regimen to recurrent UC is yet to be established. In the present case, the patient with recurrent UTUC in the contralateral ureter was initially rechallenged with the same dose and course of pembrolizumab, but only achieved partial response. However, the recurrent tumor was completely disappeared after the third cycle of treatment with a combination of pembrolizumab and erdafitinib (Figure 3), and the patient remained CR as of the final draft of the report. To the best of our knowledge, our report presented the unique case on the long-term outcomes and responses to retreatment with a combination of ICI and erdafitinib in patient with recurrent UTUC based on patient's genetic profiling. The results demonstrated a long-lasting response to treatment with 6 cycles of pembrolizumab, and a dramatic response to the ICI retreatment combined with erdafitinib in patient with advanced and recurrent UTUC harboring FGFR3 activating mutations and high TMB score. How the FGFR3 activating mutation affects the response of UTUC to ICIs may worth further mechanistic investigation.

In conclusion, genetic profiling and PD-L1 expression may provide clinicians with helpful guidance in postoperative treatment and/or retreatment in patients with UTUC treated with either targeted medicine or ICIs. Patients may obtain long-lasting responses to ICI treatment, and dramatic response to retreatment of ICIs could be achieved if the tumor recurs. However, the present report is only one case and limited in sample size. More patients with advanced metastatic UTUC treated with ICIs would be needed to warrant a satisfactory clinical trial to draw definitive conclusions.

\section{Abbreviations}

UC, urothelial carcinoma; UTUC, upper tract urothelial carcinoma; ICI, immune checkpoint inhibitor; PD-L1, programmed cell death ligand 1; $\mathrm{CR}$, complete response; TMB, tumor mutation burden; bTMB, blood tumor mutation burden; IHC, immunohistochemistry; NGS, nextgeneration sequencing; ctDNA, circulating tumor DNA; $\mathrm{CT}$, computerized tomography.

\section{Data Sharing Statement}

All data generated or analyzed during this study are included in this published article and its Supplementary Information files.

\section{Ethics Approval and Consent to Participate}

Written informed consent was obtained from the patient in accordance with the Declaration of Helsinki for the purpose of publication of the present case report and any relevant images. 


\section{Consent for Publication}

Written informed consent was obtained from the patient for publication of the case report and the accompanying images.

\section{Acknowledgments}

We would like to thank Dr. Tim Lilburn for critical reading of the manuscript.

\section{Author Contributions}

All authors made substantial contributions to conception and design, acquisition of data, or analysis and interpretation of data; took part in drafting the article or revising it critically for important intellectual content; agreed to submit to the current journal; gave final approval of the version to be published; and agree to be accountable for all aspects of the work.

\section{Funding}

This work is supported by an Innovation Grant for Science and Technology from the Dalian Government to YD for design and development of targeted sequencing panels and funding from the Affiliated Zhongshan Hospital of Dalian University to RYW for conducting the experiment.

\section{Disclosure}

The authors declare that they have no competing interests.

\section{References}

1. Babjuk M, Burger $M$, Zigeuner $R$, et al. EAU guidelines on non-muscle-invasive urothelial carcinoma of the bladder: update 2013. Eur Urol. 2013;64:639-653. doi:10.1016/j.eururo.2013.06.003

2. Munoz JJ, Ellison LM. Upper tract urothelial neoplasms: incidence and survival during the last 2 decades. $J$ Urol. 2000;164:1523-1525. doi:10.1097/00005392-200011000-00014

3. Cohen JL, Peyronnet B, Seisen T, Soukup V. SEER cancer statistics factsheets: bladder cancer. Available from: http://seer.cancer.gov/stat facts/html/urinb.html. Accessed June 12, 2020.

4. Miller NJ, Khaki AR, Diamantopoulos LN, et al. Histological subtypes and response to PD-1/PD-L1 blockade in advanced urothelial cancer: a retrospective study. $J$ Urol. 2020;204:63-70. doi:10.1097/ JU.0000000000000761

5. Kaag MG, O'Malley RL, O'Malley P, et al. Changes in renal function following nephroureterectomy may affect the use of perioperative chemotherapy. Eur Urol. 2010;58:581-587. doi:10.1016/j. eururo.2010.06.029

6. Bajorin DF, Dodd PM, Mazumdar M, et al. Long-term survival in metastatic transitional-cell carcinoma and prognostic factors predicting outcome of therapy. J Clin Oncol. 1999;17:3173-3181. doi:10.1200/ jco.1999.17.10.3173
7. Hall MC, Womack S, Roehrborn CG, Carmody T, Erickstad MD, Roehrborn CG. Prognostic factors, recurrence, and survival in transitional cell carcinoma of the upper urinary tract: a 30-year experience in 252 patients. Urology. 1998;52:594-601. doi:10.1016/s00904295(98)00295-7

8. Kamai T, Shirataki H, Nakanishi K, et al. Increased Rac1 activity and Pak1 overexpression are associated with lymphovascular invasion and lymph node metastasis of upper urinary tract cancer. $B M C$ Cancer. 2010;10:164. doi:10.1186/1471-2407-10-164

9. Yu X, Meng X, Xu M, et al. Celastrol ameliorates cisplatin nephrotoxicity by inhibiting NF-kappaB and improving mitochondrial function. EBioMedicine. 2018;36:266-280. doi:10.1016/j.ebiom.2018.09.031

10. Alavi SE, Muflih A, Akbarzadeh A, Akbarzadeh A. Cisplatin-loaded polybutylcyanoacrylate nanoparticles with improved properties as an anticancer agent. Int J Mol Sci. 2019;20:1531. doi:10.3390/ ijms20071531

11. Sonpavde G, Watson D, Tourtellott M, et al. Administration of cisplatin-based chemotherapy for advanced urothelial carcinoma in the community. Clin Genitourin Cancer. 2012;10:1-5. doi:10.1016/j. clgc.2011.11.005

12. Sonpavde G, Galsky MD, Latini D. Cisplatinineligibleandchemotherapy-ineligible patients should be the focus of new drug development in patients with advanced bladder cancer. Clin Genitourin Cancer. 2014;12:71-73. doi:10.1016/j.clgc.2013.11.016

13. Pardoll DM. The blockade of immune checkpoints in cancer immunotherapy. Nat Rev Cancer. 2012;12:252-264. doi:10.1038/ nrc3239

14. Rosenberg JE, Hoffman-Censits J, Necchi A, et al. Atezolizumab in patients with locally advanced and metastatic urothelial carcinoma who have progressed following treatment with platinum-based chemotherapy: a single-arm, multicentre, phase 2 trial. Lancet. 2016;387:1909-1920. doi:10.1016/s0140-6736(16)00561-4

15. Massard C, Gordon MS, Sharma S, et al. Safety and efficacy of durvalumab (MEDI4736), an anti-programmed cell death ligand-1 immune checkpoint inhibitor, in patients with advanced urothelial bladder cancer. J Clin Oncol. 2016;34:3119-3125. doi:10.1200/ jco.2016.67.9761

16. Balar AV, Castellano D, O’Donnell PH, et al. First-line pembrolizumab in cisplatin-ineligible patients with locally advanced and unresectable or metastatic urothelial cancer (KEYNOTE-052): a multicentre, single-arm, phase 2 study. Lancet Oncol. 2017;18:1483-1492. doi:10.1016/s1470-2045(17)30616-2

17. Balar AV, Galsky MD, Rosenberg JE, et al. Atezolizumab as first-line treatment in cisplatin-ineligible patients with locally advanced and metastatic urothelial carcinoma: a single-arm, multicentre, phase 2 trial. Lancet (London, England). 2017;389:67-76. doi:10.1016/ s0140-6736(16)32455-2

18. Tate JG, Bamford S, Jubb C, et al. COSMIC: the catalogue of somatic mutations in cancer. Nucl Acids Res. 2019;47:D941-47. doi:10.1093/nar/gky1015

19. Palma N, Morris JC, Pal SK, Ross JS, Pal SK. Exceptional response to pazopanib in a patient with urothelial carcinoma harboring FGFR3 activating mutation and amplification. Eur Urol. 2015;68:168-170. doi:10.1016/j.eururo.2015.02.023

20. Loriot Y, Necchi A, Park SH, et al. Erdafitinib in locally advanced or metastatic urothelial carcinoma. N Engl J Med. 2019;381:338-348. doi:10.1056/NEJMoa1817323

21. Warner AB, Palmer JS, Shoushtari AN, et al. Long-term outcomes and responses to retreatment in patients with melanoma treated with PD-1 blockade. J Clin Oncol. 2020;38:1655-1663. doi:10.1200/ jco.19.01464 


\section{Publish your work in this journal}

OncoTargets and Therapy is an international, peer-reviewed, open access journal focusing on the pathological basis of all cancers, potential targets for therapy and treatment protocols employed to improve the management of cancer patients. The journal also focuses on the impact of management programs and new therapeutic

Submit your manuscript here: https://www.dovepress.com/oncotargets-and-therapy-journal agents and protocols on patient perspectives such as quality of life, adherence and satisfaction. The manuscript management system is completely online and includes a very quick and fair peer-review system, which is all easy to use. Visit http://www.dovepress.com/ testimonials.php to read real quotes from published authors. 